\title{
Returning to standard surgical practice during the COVID-19 pandemic
}

\author{
Florence Lawrie $^{1 *}$, MB, BS (UK), LMCHK, Claire Nestor², MB, BCh, FCAI \\ ${ }^{1}$ Department of Anaesthesiology, Queen Mary Hospital, Hong Kong \\ ${ }^{2}$ Department of Anaesthesiology, The University of Hong Kong, Hong Kong \\ *Corresponding author: f.lawrie@mac.com
}

Hong Kong Med J 2021;27:150-1

https://doi.org/10.12809/hkmj208760

After the first confirmed case of coronavirus disease 2019 (COVID-19) in Hong Kong in January $2020,{ }^{1}$ rapid implementation of border restrictions, isolation, quarantine, and community measures such as social distancing, closure of schools, and use of face masks, facilitated the initial containment of the disease. ${ }^{2}$ Urgent and emergency surgeries have continued uninterrupted throughout the COVID-19 pandemic. However, during subsequent peaks in the spread of COVID-19, elective surgeries have been disrupted, even for asymptomatic patients with a negative history of fever, travel, occupational exposure, contacts, or clusters. This interruption of elective surgeries has had a significant and lasting impact on many patients' quality of life, in addition to lost training opportunities for anaesthetic and surgical trainees.

As a result of the many aerosol-generating procedures undertaken by our specialty, anaesthetic practice has been significantly impacted by COVID-19. In many centres, supraglottic airway devices have been abandoned and modified rapid sequence induction with tracheal intubation using videolaryngoscopy is the standard for every patient. The wearing of high-level personal protective equipment (PPE)-including N95 respirators, eye protection, gowns, gloves and caps-by the anaesthetist and the assistant(s) has been adopted during all aerosol-generating procedures. It is recommended that airborne precautions should continue to be worn by all in the room until air exchange has sufficiently reduced the airborne viral load, estimated to take 12 minutes in a well-ventilated operating room. In practice, many members of the operating team return to the room wearing lowerlevel PPE (surgical mask only) before this time. ${ }^{3}$

While we recognise the challenges in forward planning during a global pandemic, it is important to consider approaches to resume elective surgical services in the safest possible way. There are several barriers to resumption of full elective services in Hong Kong, including consumption of limited stocks of PPE, availability of in-patient bed space, patient reluctance for elective hospital admission, and the potential need for anaesthetic staff redeployment. A major concern for staff and patients alike is the inadvertent elective admission of a COVID-19 positive patient, with the potential for transmission to staff and other vulnerable patients. In order to minimise this risk, effective and reliable preadmission screening should be introduced. This has proven a significant challenge as a result of the highly variable incubation period, and the low sensitivity of testing early in the disease course. ${ }^{4}$

As different countries move towards the recovery phase, a number of approaches have been proposed, which vary according to the perceived risk of community transmission. The approaches can be divided into: pre-admission history and examination without laboratory investigation (as recommended by the Australian Commission on Safety and Quality in Health Care ${ }^{5}$ ); pre-admission with reverse transcription-polymerase chain reaction (RT-PCR) testing for COVID-19 (as recommended by American Society of Anesthesiologists ${ }^{6}$ ), or a combination of pre-admission quarantine and RT-PCR testing prior to admission (as introduced in England ${ }^{7}$ and Ireland $\left.^{8}\right)$. Although the latter is the safest and most cautious approach, it presents a number of further problems, including limited testing availability, patients' reluctance to self-isolate for social or economic reasons, and the difficulty of healthcare institutions in governing such instructions.

Advantages of pre-admission RT-PCR testing include the ability to identify asymptomatic carriers-estimated to be $43 \%$ of infected patientsand the potential to reduce community spread. ${ }^{9}$ A recent case at a public hospital in Hong Kong involved a patient admitted to a surgical ward with acute appendicitis, who was not confirmed to have COVID-19 until the sixth day of admission. Although no staff were considered close contacts, patients who had shared the same bay were traced and tested, and one other patient was subsequently diagnosed. ${ }^{10}$ Although this was an emergency case, COVID-19 testing on admission could have led to an earlier diagnosis of one or both patients, so that appropriate perioperative measures could have been arranged and staff members who had contact with the patients could have been spared significant anxiety. 
Clear guidance regarding the perioperative investigation and care of all surgical patients will bring greater clarity and reassurance to hospital staff involved in their care. The implementation of a perioperative pathway will require collaboration between all relevant stakeholders, updated regularly as the situation evolves.

\section{Author contributions}

All authors contributed to the concept or design of the study, acquisition of the data, analysis or interpretation of the data, drafting of the manuscript, and critical revision of the manuscript for important intellectual content. All authors had full access to the data, contributed to the study, approved the final version for publication, and take responsibility for its accuracy and integrity.

\section{Conflicts of interest}

The authors have disclosed no conflicts of interest.

\section{Acknowledgement}

The authors thank Prof Michael Irwin for his contribution to the concept of the study and critical revision of the manuscript for important intellectual content. As an editor of the journal, Prof Irwin was not involved in the review process for this article.

\section{Funding/support}

This commentary received no specific grant from any funding agency in the public, commercial, or not-for-profit sectors.

\section{References}

1. Centre for Health Protection, Department of Health, Hong Kong SAR Government. Latest situation of cases of COVID-19. Available from: https://www.chp.gov.hk/files/ pdf/local_situation_covid19_en.pdf. Accessed 28 Jul 2020.

2. Cowling BJ, Ali ST, Ng TW, et al. Impact assessment of non-pharmaceutical interventions against coronavirus disease 2019 and influenza in Hong Kong: an observational study. Lancet Public Health 2020;5:e279-88.

3. Cook TM. Personal protective equipment during the coronavirus disease (COVID) 2019 pandemic-a narrative review. Anaesthesia 2020;75:920-7.

4. Lother SA. Preoperative SARS-CoV-2 screening: Can it really rule out COVID-19?. Can J Anaesth 2020 Jun 23. Epub ahead of print.

5. Australian Commission on Safety and Quality in Healthcare, Australian Government. FAQ for consumers on elective surgery. Available from: https://www.safetyandquality.gov. au/faqs-consumers-elective-surgery. Accessed 27 Jul 2020.

6. The American Society of Anesthesiologists. The ASA and APSF Joint Statement on Perioperative Testing for the COVID-19 Virus. Available from: https://www.asahq.org/ about-asa/newsroom/news-releases/2020/06/asa-andapsf-joint-statement-on-perioperative-testing-for-thecovid-19-virus. Accessed 28 Jul 2020.

7. National Health Service, UK Government. Operating framework for urgent and planned services in hospital settings during COVID-19. Version 1. 14 May 2020. Available from: https://www.england.nhs.uk/coronavirus/ wp-content/uploads/sites/52/2020/05/Operatingframework-for-urgent-and-planned-services-withinhospitals.pdf. Accessed 29 Jul 2020.

8. Health Information and Quality Authority. Evidence Summary for care pathways support for the resumption of scheduled hospital care in the context of COVID-19. 18 June 2020. Available from: https://www.hiqa.ie/sites/ default/files/2020-06/Pathways-for-the-resumption-ofhospital-care-after-COVID-19.pdf. Accessed 28 Jul 2020.

9. Gudbjartsson DF, Helgason A, Jonsson H, et al. Spread of SARS-CoV-2 in the Icelandic Population. N Engl J Med 2020;382:2302-15.

10. Hong Kong SAR Government. Press release-updates on COVID-19 in Hong Kong East Cluster. Available from: https://www.info.gov.hk/gia/general/202007/21/ P2020072100655.htm. Accessed 28 Jul 2020. 